\title{
Spectral-texture approach to hyperspectral image analysis for plant classification with SVMs
}

DOI:

10.1109/IST.2017.8261496

\section{Document Version}

Accepted author manuscript

Link to publication record in Manchester Research Explorer

\section{Citation for published version (APA):}

AlSuwaidi, A., Grieve, B., \& Yin, H. (2017). Spectral-texture approach to hyperspectral image analysis for plant classification with SVMs. In 2017 IEEE International Conference on Imaging Systems and Techniques (IST) (pp. 16) https://doi.org/10.1109/IST.2017.8261496

\section{Published in:}

2017 IEEE International Conference on Imaging Systems and Techniques (IST)

\section{Citing this paper}

Please note that where the full-text provided on Manchester Research Explorer is the Author Accepted Manuscript or Proof version this may differ from the final Published version. If citing, it is advised that you check and use the publisher's definitive version.

\section{General rights}

Copyright and moral rights for the publications made accessible in the Research Explorer are retained by the authors and/or other copyright owners and it is a condition of accessing publications that users recognise and abide by the legal requirements associated with these rights.

\section{Takedown policy}

If you believe that this document breaches copyright please refer to the University of Manchester's Takedown Procedures [http://man.ac.uk/04Y6Bo] or contact uml.scholarlycommunications@manchester.ac.uk providing relevant details, so we can investigate your claim.

\section{OPEN ACCESS}




\title{
Spectral-Texture Approach to Hyperspectral Image Analysis for Plant Classification with SVMs
}

\author{
Ali AlSuwaidi*, Bruce Grieve, and Hujun Yin** \\ School of Electrical and Electronic Engineering \\ The University of Manchester \\ Manchester, M13 9PL, UK \\ *ali.bghalsuwaidi@postgrad.manchester.ac.uk, **h.yin@manchester.ac.uk
}

\begin{abstract}
Numerous environmental and financial benefits of using hyperspectral imaging have driven its increased applications on studying plant conditions. This paper is concerned with the analysis of hyperspectral images for plant discrimination by means of spectral and texture analysis approaches. The main contribution of the work lies in the use of a spectral-texture analysis based on both feature selection and Markov random field model to enhance prediction performance, as compared to conventional approaches. A hyperspectral dataset on control and stressed Arabidopsis plant leaves captured by a proximal hyperspectral imaging system was used in the experiment. Texture parameters with different orders were estimated from the MRF model and two SVM settings were used in the evaluation. Experimental results showed significant improvements in classification performance of the proposed spectral-texture approach over the conventional methods.
\end{abstract}

Index Terms-feature selection; hyperspectral imaging; markov random field; spectral analysis; texture analysis.

\section{INTRODUCTION}

Hyperspectral imaging (HSI), also termed imaging spectroscopy, has triggered a considerable amount of innovative scientific quest [1]. It is a branch of multivariate imaging [2] that involves the convergence of spectroscopy (i.e. the study of spectral properties) and remote imaging (i.e. the study of optical properties) technologies. This branch of imaging has risen in importance recently due to the benefit of capturing data from the visible through to the near-infrared wavelength ranges in hundreds of narrow band spectra [3]. HSI has several applications; for instance, in medical imaging [4], chemical processes [5], industrial processes [6], and proximal and remote sensing [7], [8]. In addition, several sensor configurations have been used to obtain hyperspectral images such as point, line, area, and single shot scanning [9].

The fundamental properties of hyperspectral images are both spectral and textural in nature. The spectral information describes the entire measured spectrum, covering single or several electromagnetic regions, where each pixel (i.e. image unit element) represents a unique spectral signature [9]. This spectral signature can be a function of reflectance, absorbance, or transmittance at each wavelength. The narrowest spectral band defines the spectral resolution - that is, the ability of the system to distinguish between two adjacent spectral bands. In contrast, texture describes the variation in intensity through an image associated with many texture properties, such as orientation, depth, smoothness, coarseness etc. [10]. Furthermore, the maximum and minimum intensity values serve as the brightest and darkest pixels respectively. In addition, the smallest object that can be detected by the HSI system is called spatial resolution and it is used to determine the clarity of the image.

Most studies focus on HSI spectral analysis, though several approaches have also investigated on texture or spectraltexture properties in the last few years. The spectral approach considers the spectral information, the texture analysis considers adjacent pixels and their intensities, while the spectraltexture considers the sequence of both in the analysis. These approaches are important for several domains such as agriculture, medicine, and industry, and play a key role in many tasks, especially image classification [9], [10]. In terms of spectral analysis, spectral signatures are used for classification. Although the entire measured spectrum is necessarily relevant to the problem investigated, commonly, techniques for feature extraction and selection - the processes of reducing information dimensionality to the most discriminant features have been used to analyse hyperspectral images efficiently and effectively, thus improving the performance, and reducing the processing time [11]. In other words, only relevant information will be used in the analysis.

Texture analysis provides an important base for object description and recognition since it gives insight into texture properties of the object. The techniques for analysing texture can be broadly categorised into statistical, structural, transform-based, and model-based [10]. The statistical technique uses the distribution of grey levels to describe the properties of a texture. A co-occurrence matrix is an example of this category where the second order statistics is used to extract texture properties. The second technique describes a texture based on existing texture properties, such as lines. It should be mentioned that this technique can describe regular textures only, so its practical applications are limited. The third technique uses the characteristics of the transformed texture to determine its properties. Some common examples of this technique are the wavelet transform and the 2-D fast Fourier transform. In the last category, an empirical texture model and its parameters are used to describe a texture. The Markov random field (MRF) is a widely used example. In the spectral-texture analysis approach, the characteristics of 
both approaches are taken into account for the classification process.

This paper focuses on distinguishing plants under several stress conditions (i.e. plants that have been subjected to heat and cold) from normal plants using a spectral-texture approach (based on feature selection and MRF) and compares the results with those from existing spectral or texture approaches. The relevant features (i.e. wavelengths in this experiment) along with the texture properties (i.e. estimated MRF parameters of different orders) are used in the classification stage. The correlation-based feature selection (CFS) algorithm has been shown to be particularly powerful. Moreover, several studies published in the past have shown the MRF to be one of the most powerful models to describe textures. Furthermore, both conventional and one-class based support vector machine (SVM) classifiers are used for classification for a comparison. The results showed significant improvements in accuracy compared with when both spectral and texture approaches are used.

The remainder of the paper is organised as follows: the background is reviewed in Section II. Section III presents the imaging system, dataset and proposed spectral-texture approach. The experimental results and discussions are given in Sections IV, followed by the concluding remarks in Section V.

\section{BACKGROUND}

This section outlines the spectral and texture approaches relevant to this work. It is divided into three subsections: an overview of feature selection is presented in the first subsection; the MRF model is highlighted in the second, and the last ends with a description of conventional and one-class SVM based classifiers.

\section{A. Feature Selection}

Recent developments in HSI systems allow more data to be collected about the problem investigated. However, not all data contains information that is necessarily useful or relevant. This issue has heightened the need for an efficient and effective analysis method as a large amount of data is being recorded and it is difficult to analyse the information directly from pixel values. Feature selection is a possible solution of this issue, whereby only a subset of relevant features (i.e. wavelengths in this study) are extracted and used in the analysis (redundant and irrelevant features are discarded). This not only reduces the dimensionality of the hyperspectral images but also enhances the prediction performance, as well as reducing the required processing time.

A good review of feature selection processes, models, and algorithms can be found in [11]-[13]. In short, the $k$-th feature $\left(x_{k}\right)$ from a feature space $\boldsymbol{x}=\left\{x_{1}, \ldots, x_{n}\right\}$ for a specific dataset $\boldsymbol{X}$ can be considered as relevant, if it can be used to distinguish between two specified different classes, $Y=$ $\{y 1, y 2\}$, in $\boldsymbol{X}$. Several algorithms have been proposed in the literatures (a good list demonstrated in [13]) with the main goal of finding an optimal subset of features $S_{o}$ which maximises the evaluation criteria $J($.$) :$

$$
J\left(S_{o}\right) \geq J\left(S_{j}\right), \forall S_{o}, S_{j} \subseteq \boldsymbol{X} ; j=1,2, \ldots, s
$$

where $S_{j}$ represents the $j$-th subset of the total subsets, $S$, generated in the feature selection process.

This study is limited to the CFS feature selection algorithm since it mainly focuses on comparing the performance of conventional and one-class classifiers, not the performance of different selection algorithms. A performance comparison of several selection algorithms can be found in our previous study [13]. The CFS is used in this study due to its ability to discard irrelevant and redundant features, i.e. the wavelengths selected are consistently lower and produce a discrimination performance better than that of other selection algorithms. CFS uses Shannon's entropy $H(X)=-\sum_{i=1}^{n} P\left(x_{i}\right) \log _{2} P\left(x_{i}\right)$ and information gain $I(X, Y)=H(X)-H(X \mid Y)$ to measure the correlation between features and classes (through symmetrical uncertainty) and then uses the measured correlation to evaluate the features relevancy [14]:

$$
\text { Merit }_{S}=\frac{t\left(\overline{r_{c f}}\right)}{t+\left(t+t(t-1) \overline{r_{f f}}\right)}
$$

where Merit $_{S}$ denotes the heuristic merit of a subset containing $t$ features; $\overline{r_{c f}}$ represents the average feature-class correlation; and $\overline{r_{f f}}$ is the average feature-feature correlation. The search strategy in this algorithm is based on a best first search, and the process halts if no improvement is achieved after five consecutive iterations.

\section{B. MRF Model}

An MRF, also known as an undirected graph model [15], is an extension of the Markov chain model [16]. It generates an empirical model of each pixel in the image, based on the corresponding intensities of the neighbouring pixels. The advantages of this model over the directed graph models are: better discrimination of the normalisation process and it is more symmetric (i.e. more natural for certain domains) [15]. An example of MRF neighbour structure, as well as the equivalent parameters, is shown in Fig. 1. The order of the MRF model depends on the structure of the neighbouring pixels and can be defined as (e.g. for a first order MRF) a local interaction sum $V$ :

$$
V=\beta_{0}+\beta_{1}\left(x_{i, j-1}+x_{i, j+1}\right)+\beta_{1}\left(x_{i-1, j}+x_{i+1, j}\right)
$$

where $V$ denotes the local interaction sum and $\left\{\beta_{0}, \beta_{1}, \beta_{2}\right\}$ are the MRF parameters. The order of $V$ can be extended to second, or third, or fourth order (see Fig. 1b).

An MRF is called isotropic, if the perpendicular parameters for a specific order are the same, and anisotropic if the parameters are different. It should be mentioned that the positive parameters govern clustering in a specific direction (e.g. $\beta_{1}$ in horizontal and $\beta_{2}$ in vertical directions) while the positive and negative values of these parameters cause attraction and repulsion, respectively. 


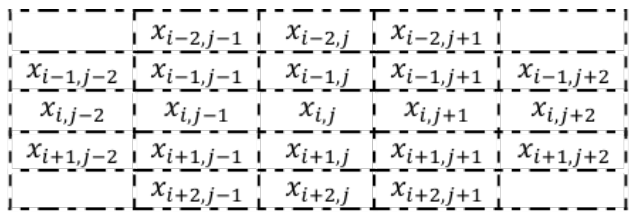

(a)

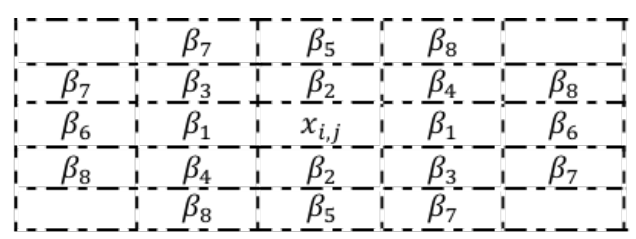

(b)

Fig. 1. Neighbourhood structure (a) and equivalent parameters (b). $\left\{\beta_{1}, \beta_{2}\right\}$ indicates first order, $\left\{\beta_{1}, \beta_{2} \beta_{3}, \beta_{4}\right\}$ represents second order, $\left\{\beta_{1}, \beta_{2} \beta_{3}, \beta_{4} \beta_{5}, \beta_{6}\right\}$ denotes third order, and $\left\{\beta_{1}, \beta_{2} \beta_{3}, \beta_{4} \beta_{5}, \beta_{6}, \beta_{7}, \beta_{8}\right\}$ indicates fourth order

Generally, the equivalent Gibbs distribution is used to describe the MRF model mathematically [17]. Gibbs distribution $P(x)$ for realisation $x$ can be represented as:

$$
P(x)=\frac{1}{Z} e^{-U(x) / T}
$$

where $T$ is a constant and stands for temperature; $U(x)$ denotes the energy function of configuration $x$; and $Z$ is a normalising constant, also termed the partition function, defined as:

$$
Z=\sum_{x} e^{-U(x) / T}
$$

Various algorithms to estimate MRF parameters have been developed over many years. Maximum likelihood (ML) and least square (LS) estimates are two popular examples. The former is a Bayesian estimate that finds a solution over a large system of nonlinear equations [18]. There is no closed form solution for the ML estimate of parameters, so an assumption about the distribution (i.e. using an alternative function) needs to be considered in this case [15]. Moreover, the result is not guaranteed in all cases and the estimation process is computationally expensive [19]. The latter estimate is very useful and easy to implement since no assumptions need to be taken into account [20]. Furthermore, the LS has lower computational requirements compared with the ML estimate. Although the estimated parameters are not as precise as the ML estimates, they are satisfactory.

In terms of estimating texture parameters, both estimates start by looking at the entire image or part of it in order to extract the parameters from the covered area. The description of the LS estimate is given in this study since it is fast and easy to implement compared with the speed and complexity of a ML estimate [19].

Let $\Omega$ denote a finite lattice and $\mathcal{N}$ represent a neighbouring system; the model parameters can then be estimated using the following equation:

$$
\hat{\beta}=\left[\sum_{m \in \Omega} \beta_{m} \beta_{m}^{\top}\right]^{-1}\left[\sum_{m \in \Omega} \sum_{i, j \in \Omega} \beta_{m} x_{i, j}\right], m=1,2, \ldots, M
$$

where $x_{i, j}$ represents the middle pixel, $\top$ stands for transpose operation, and $\beta_{m}$ denotes the neighbouring pixels that can be represented as:

$$
\beta_{m}=\operatorname{col}\left[x_{i+u, j+v}\right],(u, v) \in \mathcal{N}, \text { and }(u, v) \neq 0
$$

where $u$ and $v$ are integers (i.e. $u, v \in\left\{\mathbb{Z}^{+} \cup \mathbb{Z}^{-}\right\}$) and used to determine the location of the neighbouring pixels (i.e. horizontal and vertical) and col stands for column. It is worth noting that different MRF orders lead to a different number of parameters; two horizontal and vertical parameters in the first order, four parameters (i.e. the previous two plus two diagonal ones) in the second, and so on.

\section{Support Vector Machine}

The SVM is an example of supervised learning - i.e. learning with labels - that has a wide range of applications due to its ability to handle high dimensional data with few training samples [3]. It was originally developed to find a linear hyperplane that maximises the margin between two different classes, thus defining the optimal separable line. In the conventional case, all labels are available and well distributed. For example, the best linear hyperplane in a binary classification problem can be found by solving the following quadratic problem with the regards to a training set:

$$
\begin{aligned}
& \min _{\omega \in \mathbb{R}^{d}, \xi_{i} \in \mathbb{R}^{+}} \frac{\|\omega\|^{2}}{2}+C \sum_{i}^{N} \xi_{i} \\
& \text { subject to }: y_{i}\left(\omega \cdot \boldsymbol{x}_{\boldsymbol{i}}+b\right) \geq 1-\xi_{i}
\end{aligned}
$$

where $\|\omega\|$ is the Euclidian norm of the weighting vector; $\xi_{i}$ represents a non-zero slack variable; $y_{i} \in\{ \pm 1\}$ denotes the class label; $b$ is the bias; and $C$ stands for a regularisation constant and is used in the non-separable case to penalise the samples that lie on the other class side. In this case, both the bias and weighting vectors are used to solve the decision function $f(x)$ :

$$
f(\boldsymbol{x})=\operatorname{sign}\left(\omega \cdot \boldsymbol{x}_{\boldsymbol{i}}+b\right)
$$

where the value of the decision function lies between $\in\{ \pm 1\}$ and +1 denotes one class and -1 the other class.

On the other hand, a well-known one-class distribution is only available (i.e. unbalanced) in the unconventional case, also known as one-class or novelty detection (ND). The prediction performance is measured based on the ability of the constructed model to distinguish samples similar to those used in training, from others not presented in the training process. ND techniques can be classified into five categories: density based, distance based, reconstruction based, domain based, and information based [21]. 
In recent years, ND has received attention across a number of disciplines, and is currently employed in several domains, especially in monitoring and analysing high interiority systems; here, normal samples are the most dominant and the distributions of the abnormal classes are difficult, if not impossible, to obtain [22].

In terms of implementation with the SVM, a modification has to be made to the conventional SVM in order to deal with the unbalanced data [23]. In this case, the best hyperplane is represented as a hyperplane that separates the data from the origin with a maximum margin. Similar to conventional cases, a quadratic programming routine is used to solve the following quadratic equation:

$$
\begin{array}{r}
\min _{\omega \in \mathbb{R}^{d}, \xi_{i} \in \mathbb{R}^{+} b \in \mathbb{R}} \frac{\|\omega\|^{2}}{2}+C \sum_{i} \xi_{i}-b \\
\text { subject to }:\left(\omega \cdot \phi\left(\boldsymbol{x}_{\boldsymbol{i}}\right)\right) \geq b-\xi_{i}, \forall \xi_{i} \geq 0
\end{array}
$$

where $\phi\left(\boldsymbol{x}_{\boldsymbol{i}}\right)$ is the transformed training vector $x_{i}$. It should be mentioned that $C=\frac{1}{\vartheta}$, where $\vartheta \in\{0,1\}$ and $\vartheta$ is the lower bound of the support vectors, as well as the upper bound of the outliers, used to determine the flexibility of the decision boundary. Moreover, the decision boundary $f(\boldsymbol{x})=$ $\operatorname{sign}\left(\omega \cdot \phi\left(\boldsymbol{x}_{\boldsymbol{i}}\right)-b\right)$ values lie in $\{ \pm 1\}$ where +1 denotes normal class (i.e. within the boundary) and -1 represents the abnormal. In addition, the linear boundaries of these classifiers (i.e. conventional and ND) can be extended to non-linear cases through kernel tricks (i.e. transforming the data into a higher dimensional space). Several kernels are available, such as the radial basis function used in this study and the polynomial:

$$
\begin{array}{r}
\operatorname{RBF}: K(\boldsymbol{x}, \boldsymbol{y})=e^{-\gamma\|\boldsymbol{x}-\boldsymbol{y}\|^{2}}, \gamma=\frac{1}{2 \sigma^{2}} \\
\text { Polynomial : } K(\boldsymbol{x}, \boldsymbol{y})=((\boldsymbol{x}, \boldsymbol{y})+1)^{n}, n \in \mathbb{R}^{+}
\end{array}
$$

\section{Materials And Proposed Method}

This section describes the materials and the proposed spectral-texture approach. It first emphasises the specifications of the HSI used to capture the dataset, then describes the dataset used for the evaluation, followed by a detailed description of the proposed approach.

\section{A. HSI System and Dataset}

The HSI system used to record the images was based on a low-noise Peltier-cooled digital system (Hamamatsu, model C4742-95-12ER, Hamamatsu Photonics K.K., Hamamatsu, Japan) covering the visible wavelength range from 400 to 720 $\mathrm{nm}$, with a spectral resolution of up to $10 \mathrm{~nm}$ and a spatial resolution of $1024 \times 1344$, resulting in 33 narrow spectral bands (described in [24]). Moreover, a fast liquid crystal tunable filter and an infrared blocking filter were mounted in front of the lens to control the spectral transmission electronically, and to prevent leakage. Furthermore, the pixel intensities were recorded using a 12-bit analogue to digital converter and their responses were linear over the entire dynamic range. In addition, the images were captured in a dark room in order to minimise the effect of unwanted noise.

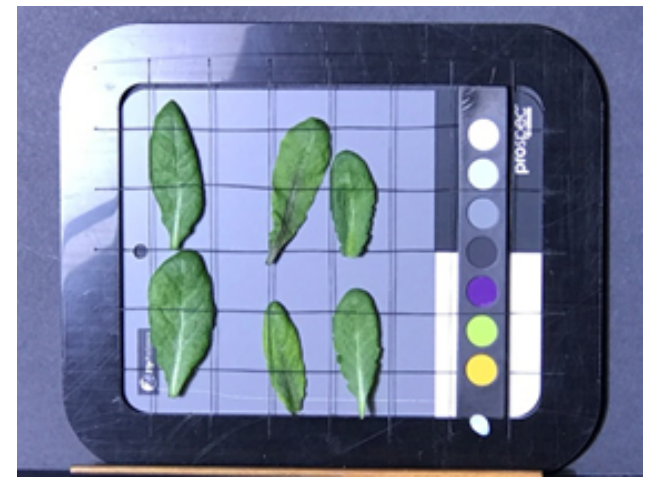

Fig. 2. Leaf samples. True colour of a scene image captured on January 2017 for illustration purpose

Three different images were captured; scene (actual image), dark noise (electronic noise), and flat field (illumination distribution) images. The scene image consisted of six Arabidopsis leaf samples placed flat on the sample plate; two of which had been subjected to normal conditions, while the other four had not (two heat and two cold-stressed). A reference colour patch was also included for further calibration. The flat field and dark noise images were used to spectrally normalise the scene image, thus enhancing the quality of the image. In the example of scene image, shown in Fig. 2, leaves on the left (top and bottom) represent the controlled leaves, while leaves in the middle and on the right (top and bottom) represent cold and heat stressed, respectively. In total, 18 replicated scene images were obtained on the same day. 648 samples were extracted from all scene images and divided into two groups: normal and stressed (i.e. abnormal in ND case). The normal group was represented by 216 samples and the remaining samples represented the stressed group. It should be stated that only green areas were considered for sample extraction (i.e. spectral signature and texture properties).

\section{B. Spectral-Texture Framework}

The following steps describe the framework of the proposed spectral-texture approach (as shown in Fig. 3). To begin with, spectral signatures are extracted from the pixel values of a small leaf region and then averaged over the entire wavelength spectrum to reduce the variation in pixel intensities across the selected leaf region. After extracting the spectral signature, the CFS algorithm is used to simplify the dataset and select the most significant wavelengths, thus projecting the data in a subspace without scarifying the wavelengths relevant to the problem. Following the selection process, the LS method is used to estimate the first, second, third, and fourth order parameters of the MRF model, which are averaged either over the entire wavelength spectrum or over the list of selected wavelengths. Finally, the selected wavelengths and the estimated texture parameters are passed on to the classifiers for the discrimination process. Eq. 9 is used in the conventional case, while the novelty score $z(\boldsymbol{x})$ can also be used for 
TABLE I

Average Classification Rate

\begin{tabular}{|c|c|c|}
\hline \multirow[t]{2}{*}{ Approach } & \multicolumn{2}{|c|}{$\begin{array}{c}\text { Average Classification Rate }(\%) \\
\text { (Standard Deviation) }\end{array}$} \\
\hline & SVM & ND-SVM \\
\hline All Wavelengths & $87.68(0.017)$ & $86.00(0.020)$ \\
\hline CFS & $88.40(0.014)$ & $89.95(0.015)$ \\
\hline Texture, $1^{\text {st }}$ order & $54.47(0.022)$ & $53.51(0.029)$ \\
\hline Texture, $2^{\text {nd }}$ order & $71.94(0.019)$ & $65.56(0.029)$ \\
\hline Texture, $3^{\text {rd }}$ order & $78.27(0.016)$ & $66.38(0.039)$ \\
\hline Texture, $4^{\text {th }}$ order & $80.63(0.017)$ & $63.86(0.038)$ \\
\hline All Wavelengths+Texture, $1^{\text {st }}$ order & $85.36(0.018)$ & $87.22(0.020)$ \\
\hline All Wavelengths+Texture, $2^{\text {nd }}$ order & $83.42(0.017)$ & $87.85(0.019)$ \\
\hline All Wavelengths+Texture, $3^{\text {rd }}$ order & $80.04(0.018)$ & $87.65(0.020)$ \\
\hline All Wavelengths+Texture, $4^{\text {th }}$ order & $77.23(0.015)$ & $89.11(0.017)$ \\
\hline CFS+Texture, $1^{\text {st }}$ order (all) & $90.19(0.012)$ & $91.57(0.014)$ \\
\hline CFS+Texture, $2^{\text {nd }}$ order (all) & $93.14(0.010)$ & $93.36(0.016)$ \\
\hline CFS+Texture, $3^{\text {rd }}$ order (all) & $92.87(0.011)$ & $92.29(0.021)$ \\
\hline CFS+Texture, $4^{\text {th }}$ order (all) & $91.97(0.013)$ & $91.83(0.018)$ \\
\hline CFS+Texture, $1^{\text {st }}$ order (FS) & $88.65(0.014)$ & $90.27(0.014)$ \\
\hline CFS+Texture, $2^{\text {nd }}$ order (FS) & $88.53(0.014)$ & $90.07(0.016)$ \\
\hline CFS+Texture, $3^{\text {rd }}$ order (FS) & $88.37(0.012)$ & $90.31(0.021)$ \\
\hline CFS+Texture, $4^{\text {th }}$ order (FS) & $88.33(0.015)$ & $90.00(0.018)$ \\
\hline
\end{tabular}

discrimination:

$$
z(\boldsymbol{x})=b-\omega \cdot \phi\left(\boldsymbol{x}_{\boldsymbol{i}}\right)
$$

where $\phi\left(\boldsymbol{x}_{\boldsymbol{i}}\right)$ is the transformed vector $x_{i}$ using RBF kernel.

\section{RESUlts AND Discussions}

The experiment assessed the utility of the proposed approach for analysing and classifying plant hyperspectral datasets under stress conditions. Several parameters (i.e. estimated from the MRF model) were used with the conventional and ND classifiers to generate the results, thus finding the optimal order. The final results were then compared with those from existing conventional approaches. It should be stated that no comparison between the selection algorithm and existing empirical indices (i.e. vegetation and disease) in the literature was performed since this comparison was already demonstrated in our previous work [13]. In addition, $50 \%$ of the samples were used for training with 10-fold cross validation and the remaining samples for testing in the conventional SVM classifier, while $60 \%$ of the normal samples were used for training and the remaining $40 \%$ and all the abnormal samples were used for testing and validation. The mean classification rate of 100 runs with the standard deviation of the conventional and non-conventional classifiers is illustrated in Table I.

Closer inspection of Table I shows the advantage of the proposed framework when compared with existing conventional approaches. The classification rate of the proposed framework displayed an improvement of $4.7 \%$ and $3.4 \%$ for conventional and ND classifiers respectively compared with conventional approaches. Moreover, the estimated parameters from the second order MRF tended to be more powerful compared with the estimated ones from other orders, thus outperforming the prediction performance. In addition, the prediction performance following the proposed approach of both classifiers were almost the same (i.e. slight improvement in ND case). This highlighted the utility of the latter setting since only the normal class were used while both classes were used in the latter. It should be mentioned that, the performance of the average parameters over the entire wavelengths outperformed the one with the mean of the selected ones using current dataset, while it was not the same using another dataset (latter outperformed the former and the result is not included in this paper).

\section{CONCLusions}

This paper presents a spectral-texture analysis based approach for classifying hyperspectral plants under stress conditions. The experimental results revealed that improvements in discrimination performance of the proposed approach were achieved as compared to the conventional analysis and approaches. Moreover, the use of the second order estimated parameters combined with the spectral signature (i.e. selected wavelengths) showed marked improvements in discrimination performance compared with those of other orders. Further statistical tests showed a significant improvement of the proposed approach compared to other approaches, at a significance level of $1 \%$. In addition, a slight improvement was demonstrated with the use of non-conventional SVM (i.e. novelty detection (ND)) compared to the use of the conventional SVM; the benefit of the ND is that only the normal samples are needed and used. This means that although the conventional SVM can play an important role, the features may not be applicable to other plant stress conditions beyond the data used in this work. In contrast, the ND based SVM setting may not be affected (or only slightly) since only the normal samples are used for the construction of the classification model. Ultimately, the findings suggest the proposed approach is useful for detecting stress in plants in either balanced or unbalanced data cases. Future work will explore the use of the proposed approach on different hyperspectral datasets (e.g. with different types and conditions) and the use of other parameter estimates (e.g. ML) to further enhance the identification process.

\section{ACKNOWLEDGMENT}

The authors would like to thank Dr. Giles Johnson and Miss Helena Herrmann for the provision of Arabidopsis plants and Dr. Kinjiro Amano for helping with the data collection from the HSI system.

\section{REFERENCES}

[1] A. F. Goetz, G. Vane, J. E. Solomon, and B. N. Rock, "Imaging spectrometry for earth remote sensing," Science, vol. 228, no. 4704, pp. 1147-1153, 1985.

[2] P. L. M. Geladi, H. F. Grahn, and J. E. Burger, "Multivariate images, hyperspectral imaging: Background and equipment," in Techniques and Applications of Hyperspectral Image Analysis. John Wiley and Sons, Ltd, 2007, pp. 1-15. 


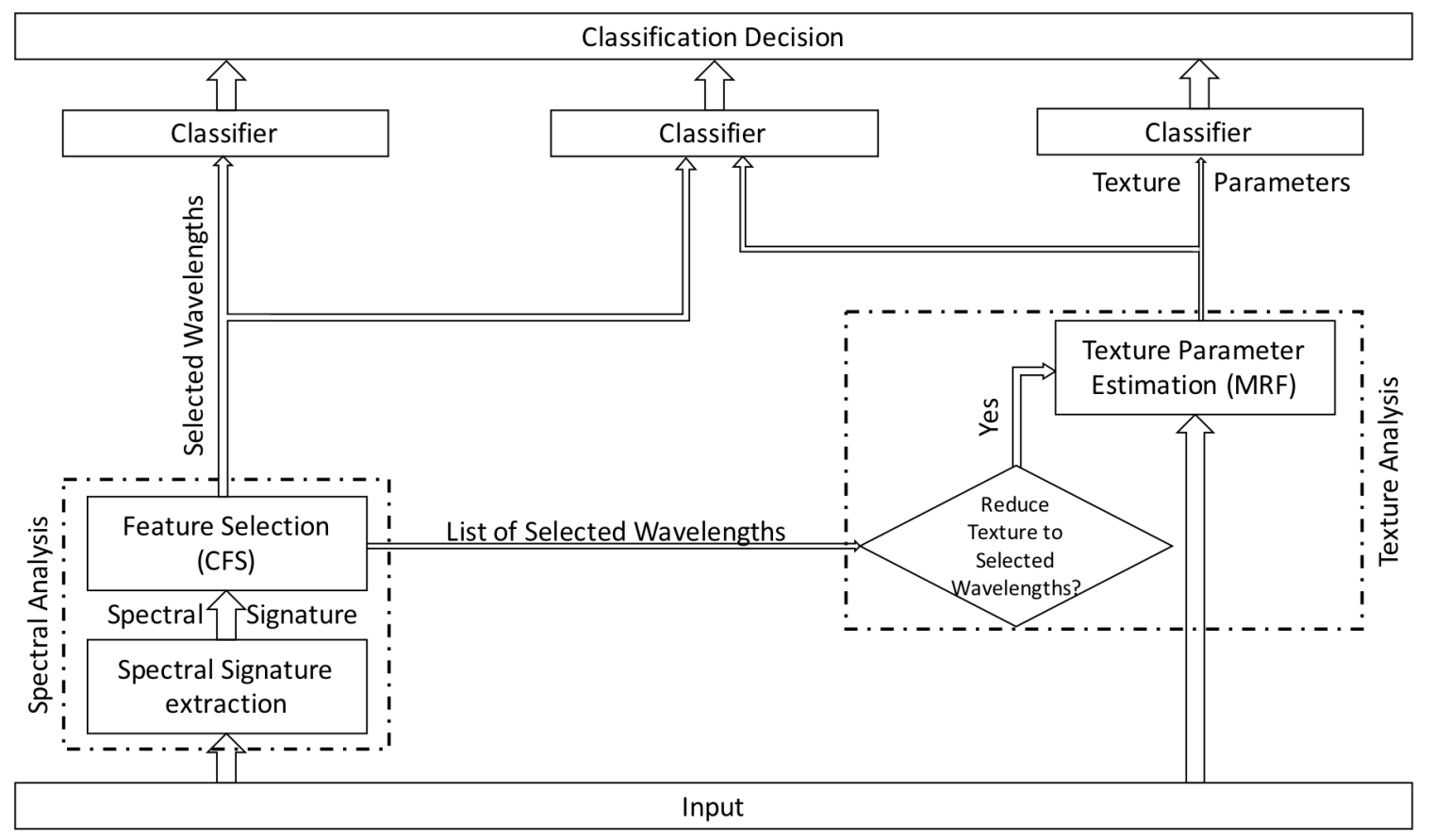

Fig. 3. Schematic of the proposed spectral-texture approach

[3] P. Ghamisi, J. Plaza, Y. Chen, J. Li, and A. J. Plaza, "Advanced spectral classifiers for hyperspectral images: A review," IEEE Geoscience and Remote Sensing Magazine, vol. 5, no. 1, pp. 8-32, March 2017.

[4] G. Lu and B. Fei, "Medical hyperspectral imaging: a review," Journal of Biomedical Optics, vol. 19, no. 1, p. 010901, 2014.

[5] P. Geladi, E. Bengtsson, K. Esbensen, and H. Grahn, "Image analysis in chemistry i. properties of images, greylevel operations, the multivariate image," TrAC Trends in Analytical Chemistry, vol. 11, no. 1, pp. 41 53, 1992.

[6] C. Duchesne, J. Liu, and J. MacGregor, "Multivariate image analysis in the process industries: A review," Chemometrics and Intelligent Laboratory Systems, vol. 117, pp. 116 - 128, 2012.

[7] H. Liu, S.-H. Lee, and J. S. Chahl, "Development of a proximal machine vision system for off-season weed mapping in broadacre no-tillage fallows," Journal of Computer Science, vol. 9, no. 12, pp. 1803-1821, 213.

[8] J. Campbell and R. Wynne, Introduction to Remote Sensing, 5th ed. Guilford Publications, 2011.

[9] G. ElMasry and D.-W. Sun, "Chapter 1 - principles of hyperspectral imaging technology," in Hyperspectral Imaging for Food Quality Analysis and Control, D.-W. Sun, Ed. San Diego: Academic Press, 2010, pp. $3-43$.

[10] M. H. Bharati, J. Liu, and J. F. MacGregor, "Image texture analysis: methods and comparisons," Chemometrics and Intelligent Laboratory Systems, vol. 72, no. 1, pp. $57-71,2004$.

[11] H. Liu and H. Motoda, Feature Selection for Knowledge Discovery and Data Mining. Norwell, MA, USA: Kluwer Academic Publishers, 1998.

[12] H. Liu and L. Yu, "Toward integrating feature selection algorithms for classification and clustering," IEEE Transactions on Knowledge and Data Engineering, vol. 17, no. 4, pp. 491-502, April 2005.

[13] A. AlSuwaidi, C. Veys, M. Hussey, B. Grieve, and H. Yin, "Hyperspectral selection based algorithm for plant classification," in 2016 IEEE International Conference on Imaging Systems and Techniques (IST), Oct 2016, pp. 395-400.

[14] M. A. Hall and L. A. Smith, "Feature selection for machine learning: Comparing a correlation-based filter approach to the wrapper," in Proceedings of the Twelfth International Florida Artificial Intelligence Research Society Conference, 1999, pp. 235-239.

[15] K. P. Murphy, Machine Learning: A Probabilistic Perspective. The MIT Press, 2012.
[16] A. Blake, P. Kohli, and C. Rother, Markov Random Fields for Vision and Image Processing. The MIT Press, 2011.

[17] S. Geman and D. Geman, "Stochastic relaxation, gibbs distributions, and the bayesian restoration of images," IEEE Transactions on Pattern Analysis and Machine Intelligence, vol. PAMI-6, no. 6, pp. 721-741, Nov 1984.

[18] C. F. Borges, "On the estimation of markov random field parameters," IEEE Transactions on Pattern Analysis and Machine Intelligence, vol. 21, no. 3, pp. 216-224, Mar 1999.

[19] H. Yin and N. M. Allinson, "Self-organised parameter estimation and segmentation of mrf model-based texture images," in Image Processing, 1994. Proceedings. ICIP-94., IEEE International Conference, vol. 2. IEEE, 1994, pp. 645-649.

[20] S. Rogers and M. Girolami, A First Course in Machine Learning, 1st ed. Chapman \& Hall/CRC, 2011.

[21] M. A. Pimentel, D. A. Clifton, L. Clifton, and L. Tarassenko, "A review of novelty detection," Signal Processing, vol. 99, pp. 215 - 249, 2014.

[22] D. A. Clifton, L. A. Clifton, P. R. Bannister, and L. Tarassenko, Automated Novelty Detection in Industrial Systems. Berlin, Heidelberg: Springer Berlin Heidelberg, 2008, pp. 269-296.

[23] B. Schölkopf, R. Williamson, A. Smola, J. Shawe-Taylor, and J. Platt, "Support vector method for novelty detection," in Proceedings of the 12th International Conference on Neural Information Processing Systems, ser. NIPS'99. Cambridge, MA, USA: MIT Press, 1999, pp. $582-588$.

[24] D. H. Foster, K. Amano, and S. M. C. Nascimento, "Color constancy in natural scenes explained by global image statistics," Visual Neuroscience, vol. 23, no. 3-4, p. 341349, 2006. 